\title{
Correlation functions evolution for the Glauber dynamics in continuum
}

\author{
Dmitri Finkelshtein* $\quad$ Yuri Kondratiev ${ }^{\dagger} \quad$ Oleksandr Kutoviy $^{\ddagger}$
}

September 28, 2018

\begin{abstract}
We construct a correlation functions evolution corresponding to the Glauber dynamics in continuum. Existence of the corresponding strongly continuous contraction semigroup in a proper Banach space is shown. Additionally we prove the existence of the evolution of states and study their ergodic properties.
\end{abstract}

\section{Introduction}

Among all birth-and-death Markov processes on configuration spaces in continuum the Glauber type stochastic dynamics is the object of permanent interest for discovering. These dynamics have the given reversible states which are grand canonical Gibbs measures. This gives a standard way to construct properly associated stationary Markov processes using the corresponding (nonlocal) Dirichlet forms related to the considered Markov generators and Gibbs measures. These processes describe the equilibrium Glauber dynamics which preserve the initial Gibbs state in the time evolution, see, e.g., [13, [14, 15], 6. Note that, in applications, the time evolution of initial state is the subject of the primary interest. Therefore, we understand the considered stochastic (nonequilibrium) dynamics as the evolution of initial distributions for the system. Actually, the Markov process (provided it exists) itself gives a general technical equipment to study this problem. However, we note that the transition from the micro-state evolution corresponding to the given initial configuration to the macro-state dynamics is the well developed concept in the theory of infinite particle systems. This point of view appeared initially in the framework of the Hamiltonian dynamics of classical gases, see, e.g., [2].

\footnotetext{
*Institute of Mathematics, National Academy of Sciences of Ukraine, Kyiv, Ukraine (fdl@imath.kiev.ua).

${ }^{\dagger}$ Fakultät für Mathematik, Universität Bielefeld, 33615 Bielefeld, Germany (kondrat@math.uni-bielefeld.de)

${ }^{\ddagger}$ Fakultät für Mathematik, Universität Bielefeld, 33615 Bielefeld, Germany (kutoviy@math.uni-bielefeld.de).
} 
The study of the non-equilibrium Glauber dynamics needs construction of the time evolution for a wider class of initial measures. The lack of the general Markov processes techniques for the considered systems makes necessary to develop alternative approaches to study the state evolutions in the Glauber dynamics. The approach realized in [11, [12, [5] is probably the only known at the present time. The description of the time evolutions for measures on configuration spaces in terms of an infinite system of evolutional equations for the corresponding correlation functions was used there. The latter system is a Glauber evolution's analog of the famous BBGKY-hierarchy for the Hamiltonian dynamics.

Here we extend constructive approach developed in [5] to correlation function evolution of the Glauber dynamics in continuum. We describe a reasonable Banach space where the evolution problem can be solved. Moreover, we construct an explicit approximation by bounded operators of the corresponding evolutional semigroup. We prove that functions in this evolution stay correlation functions of some measures (states) on configuration spaces; this means that we show the existence of states evolution. At the end we obtain the ergodic properties of the state evolution.

\section{Basic facts and notation}

Let $\mathcal{B}\left(\mathbb{R}^{d}\right)$ be the family of all Borel sets in $\mathbb{R}^{d}, d \geq 1 ; \mathcal{B}_{\mathrm{b}}\left(\mathbb{R}^{d}\right)$ denote the system of all bounded sets in $\mathcal{B}\left(\mathbb{R}^{d}\right)$.

The configuration space over space $\mathbb{R}^{d}$ consists of all locally finite subsets (configurations) of $\mathbb{R}^{d}$, namely,

$$
\Gamma=\Gamma_{\mathbb{R}^{d}}:=\left\{\gamma \subset \mathbb{R}^{d}|| \gamma \cap \Lambda \mid<\infty, \text { for all } \Lambda \in \mathcal{B}_{\mathrm{b}}\left(\mathbb{R}^{d}\right)\right\} .
$$

The space $\Gamma$ is equipped with the vague topology, i.e., the minimal topology for which all mappings $\Gamma \ni \gamma \mapsto \sum_{x \in \gamma} f(x) \in \mathbb{R}$ are continuous for any continuous function $f$ on $\mathbb{R}^{d}$ with compact support; note that the summation in $\sum_{x \in \gamma} f(x)$ is taken over only finitely many points of $\gamma$ which belong to the support of $f$. In [10, it was shown that $\Gamma$ with the vague topology may be metrizable and it becomes a Polish space (i.e., complete separable metric space). Corresponding to this topology, Borel $\sigma$-algebra $\mathcal{B}(\Gamma)$ is the smallest $\sigma$-algebra for which all mappings $\Gamma \ni \gamma \mapsto\left|\gamma_{\Lambda}\right| \in \mathbb{N}_{0}:=\mathbb{N} \cup\{0\}$ are measurable for any $\Lambda \in \mathcal{B}_{\mathrm{b}}\left(\mathbb{R}^{d}\right)$. Here $\gamma_{\Lambda}:=\gamma \cap \Lambda$, and $|\cdot|$ means the cardinality of a finite set.

The space of $n$-point configurations in an arbitrary $Y \in \mathcal{B}\left(\mathbb{R}^{d}\right)$ is defined by

$$
\Gamma_{Y}^{(n)}:=\{\eta \subset Y|| \eta \mid=n\}, \quad n \in \mathbb{N} .
$$

We set also $\Gamma_{Y}^{(0)}:=\{\emptyset\}$. As a set, $\Gamma_{Y}^{(n)}$ may be identify with the symmetrization of

$$
\widetilde{Y^{n}}=\left\{\left(x_{1}, \ldots, x_{n}\right) \in Y^{n} \mid x_{k} \neq x_{l} \text { if } k \neq l\right\} .
$$


Hence one can introduce the corresponding Borel $\sigma$-algebra, which we denote by $\mathcal{B}\left(\Gamma_{Y}^{(n)}\right)$. The space of finite configurations in an arbitrary $Y \in \mathcal{B}\left(\mathbb{R}^{d}\right)$ is defined by

$$
\Gamma_{0, Y}:=\bigsqcup_{n \in \mathbb{N}_{0}} \Gamma_{Y}^{(n)}
$$

This space is equipped with the topology of disjoint unions. Therefore, one can introduce the corresponding Borel $\sigma$-algebra $\mathcal{B}\left(\Gamma_{0, Y}\right)$. In the case of $Y=\mathbb{R}^{d}$ we will omit the index $Y$ in the notation, namely, $\Gamma_{0}:=\Gamma_{0, \mathbb{R}^{d}}, \Gamma^{(n)}:=\Gamma_{\mathbb{R}^{d}}^{(n)}$.

The restriction of the Lebesgue product measure $(d x)^{n}$ to $\left(\Gamma^{(n)}, \mathcal{B}\left(\Gamma^{(n)}\right)\right)$ we denote by $m^{(n)}$. We set $m^{(0)}:=\delta_{\{\emptyset\}}$. The Lebesgue-Poisson measure $\lambda$ on $\Gamma_{0}$ is defined by

$$
\lambda:=\sum_{n=0}^{\infty} \frac{1}{n !} m^{(n)} .
$$

For any $\Lambda \in \mathcal{B}_{\mathrm{b}}\left(\mathbb{R}^{d}\right)$ the restriction of $\lambda$ to $\Gamma_{\Lambda}:=\Gamma_{0, \Lambda}$ will be also denoted by $\lambda$. The space $(\Gamma, \mathcal{B}(\Gamma))$ is the projective limits of the family of spaces $\left\{\left(\Gamma_{\Lambda}, \mathcal{B}\left(\Gamma_{\Lambda}\right)\right)\right\}_{\Lambda \in \mathcal{B}_{\mathrm{b}}\left(\mathbb{R}^{d}\right)}$. The Poisson measure $\pi$ on $(\Gamma, \mathcal{B}(\Gamma))$ is given as the projective limit of the family of measures $\left\{\pi^{\Lambda}\right\}_{\Lambda \in \mathcal{B}_{\mathrm{b}}\left(\mathbb{R}^{d}\right)}$ where $\pi^{\Lambda}:=e^{-m(\Lambda)} \lambda$ is the probability measure on $\left(\Gamma_{\Lambda}, \mathcal{B}\left(\Gamma_{\Lambda}\right)\right)$. Here $m(\Lambda)$ is the Lebesgue measure of $\Lambda \in \mathcal{B}_{\mathrm{b}}\left(\mathbb{R}^{d}\right)$.

For any measurable function $f: \mathbb{R}^{d} \rightarrow \mathbb{R}$ we define a Lebesgue-Poisson exponent

$$
e_{\lambda}(f, \eta):=\prod_{x \in \eta} f(x), \quad \eta \in \Gamma_{0} ; \quad e_{\lambda}(f, \emptyset):=1
$$

Then, by (2.2), for $f \in L^{1}\left(\mathbb{R}^{d}, d x\right)$ we obtain $e_{\lambda}(f) \in L^{1}\left(\Gamma_{0}, d \lambda\right)$ and

$$
\int_{\Gamma_{0}} e_{\lambda}(f, \eta) d \lambda(\eta)=\exp \left\{\int_{\mathbb{R}^{d}} f(x) d x\right\} .
$$

A set $M \in \mathcal{B}\left(\Gamma_{0}\right)$ is called bounded if there exists $\Lambda \in \mathcal{B}_{\mathrm{b}}\left(\mathbb{R}^{d}\right)$ and $N \in \mathbb{N}$ such that $M \subset \bigsqcup_{n=0}^{N} \Gamma_{\Lambda}^{(n)}$. We will use the following classes of functions on $\Gamma_{0}$ : $L_{\mathrm{ls}}^{0}\left(\Gamma_{0}\right)$ is the set of all measurable functions on $\Gamma_{0}$ which have a local support, i.e. $G \in L_{\mathrm{ls}}^{0}\left(\Gamma_{0}\right)$ if there exists $\Lambda \in \mathcal{B}_{b}\left(\mathbb{R}^{d}\right)$ such that $G \uparrow_{\Gamma_{0} \backslash \Gamma_{\Lambda}}=0 ; B_{\mathrm{bs}}\left(\Gamma_{0}\right)$ is the set of bounded measurable functions with bounded support, i.e. $G \uparrow_{\Gamma_{0} \backslash B}=0$ for some bounded $B \in \mathcal{B}\left(\Gamma_{0}\right)$.

Any $\mathcal{B}\left(\Gamma_{0}\right)$-measurable function $G$ on $\Gamma_{0}$, in fact, is a sequence of functions $\left\{G^{(n)}\right\}_{n \in \mathbb{N}_{0}}$ where $G^{(n)}$ is a $\mathcal{B}\left(\Gamma^{(n)}\right)$-measurable function on $\Gamma^{(n)}$.

On $\Gamma$ we consider the set of cylinder functions $\mathcal{F}_{\text {cyl }}(\Gamma)$. These functions are characterized by the following relation: $F(\gamma)=F \Gamma_{\Gamma_{\Lambda}}\left(\gamma_{\Lambda}\right)$.

There is the following mapping from $L_{\mathrm{ls}}^{0}\left(\Gamma_{0}\right)$ into $\mathcal{F}_{\text {cyl }}(\Gamma)$, which plays the key role in our further considerations:

$$
K G(\gamma):=\sum_{\eta \Subset \gamma} G(\eta), \quad \gamma \in \Gamma,
$$


where $G \in L_{1 \mathrm{~s}}^{0}\left(\Gamma_{0}\right)$, see, e.g., [9, 16, 17]. The summation in (2.5) is taken over all finite subconfigurations $\eta \in \Gamma_{0}$ of the (infinite) configuration $\gamma \in \Gamma$; we denote this, by the symbol, $\eta \Subset \gamma$. The mapping $K$ is linear, positivity preserving, and invertible, with

$$
K^{-1} F(\eta):=\sum_{\xi \subset \eta}(-1)^{|\eta \backslash \xi|} F(\xi), \quad \eta \in \Gamma_{0} .
$$

Here and in the sequel inclusions like $\xi \subset \eta$ hold for $\xi=\emptyset$ as well as for $\xi=\eta$. We denote the restriction of $K$ onto functions on $\Gamma_{0}$ by $K_{0}$.

For any fixed $C>1$ we consider the following space of $\mathcal{B}\left(\Gamma_{0}\right)$-measurable functions

$$
\mathcal{L}_{C}:=\left\{G: \Gamma_{0} \rightarrow \mathbb{R}\left|\|G\|_{C}:=\int_{\Gamma_{0}}\right| G(\eta) \mid C^{|\eta|} d \lambda(\eta)<\infty\right\} .
$$

In the sequel, $\mathcal{L}_{C}^{\mathrm{ls}}$ denotes the set $L_{\mathrm{ls}}^{0}\left(\Gamma_{0}\right) \cap \mathcal{L}_{C}$. The space $\mathcal{L}_{C}$ can be made into a Banach space in a standard way; simply taking the quotient space with respect to the kernel of $\|\cdot\|_{C}$. To simplify notations, we use the same symbol $\mathcal{L}_{C}$ for the corresponding Banach space.

A measure $\mu \in \mathcal{M}_{\mathrm{fm}}^{1}(\Gamma)$ is called locally absolutely continuous with respect to (w.r.t. for short) Poisson measure $\pi$ if for any $\Lambda \in \mathcal{B}_{\mathrm{b}}\left(\mathbb{R}^{d}\right)$ the projection of $\mu$ onto $\Gamma_{\Lambda}$ is absolutely continuous w.r.t. projection of $\pi$ onto $\Gamma_{\Lambda}$. By [9], in this case, there exists a correlation functional $k_{\mu}: \Gamma_{0} \rightarrow \mathbb{R}_{+}$such that for any $G \in B_{\mathrm{bs}}\left(\Gamma_{0}\right)$ the following equality holds

$$
\int_{\Gamma}(K G)(\gamma) d \mu(\gamma)=\int_{\Gamma_{0}} G(\eta) k_{\mu}(\eta) d \lambda(\eta) .
$$

Restrictions $k_{\mu}^{(n)}$ of this functional on $\Gamma_{0}^{(n)}, n \in \mathbb{N}_{0}$ are called correlation functions of the measure $\mu$. Note that $k_{\mu}^{(0)}=1$.

We recall now without a proof the partial case of the well-known technical lemma (cf., [15) which plays very important role in our calculations.

Lemma 2.1. For any measurable function $H: \Gamma_{0} \times \Gamma_{0} \times \Gamma_{0} \rightarrow \mathbb{R}$

$$
\int_{\Gamma_{0}} \sum_{\xi \subset \eta} H(\xi, \eta \backslash \xi, \eta) d \lambda(\eta)=\int_{\Gamma_{0}} \int_{\Gamma_{0}} H(\xi, \eta, \eta \cup \xi) d \lambda(\xi) d \lambda(\eta)
$$

if only both sides of the equality make sense.

\section{Non-equilibrium Glauber dynamics in contin- uum}

Let $\phi: \mathbb{R}^{d} \rightarrow \mathbb{R}_{+}:=[0 ;+\infty)$ be an even non-negative function which satisfies the following integrability condition

$$
C_{\phi}:=\int_{\mathbb{R}^{d}}\left(1-e^{-\phi(x)}\right) d x<+\infty
$$


For any $\gamma \in \Gamma, x \in \mathbb{R}^{d} \backslash \gamma$ we set

$$
E^{\phi}(x, \gamma):=\sum_{y \in \gamma} \phi(x-y) \in[0 ; \infty] .
$$

Let us define the (pre-)generator of the Glauber dynamics: for any $F \in$ $\mathcal{F}_{\text {cyl }}(\Gamma)$ we set

$$
\begin{aligned}
(L F)(\gamma):= & \sum_{x \in \gamma}[F(\gamma \backslash x)-F(\gamma)] \\
& +z \int_{\mathbb{R}^{d}}[F(\gamma \cup x)-F(\gamma)] \exp \left\{-E^{\phi}(x, \gamma)\right\} d x, \quad \gamma \in \Gamma .
\end{aligned}
$$

Here $z>0$ is the activity parameter. Note that for any $F \in \mathcal{F}_{\text {cyl }}(\Gamma)$ there exists $\Lambda \in \mathcal{B}_{\mathrm{b}}\left(\mathbb{R}^{d}\right)$ such that $F(\gamma \backslash x)=F(\gamma)$ for any $x \in \gamma_{\Lambda^{c}}$ and $F(\gamma \cup x)=F(\gamma)$ for any $x \in \Lambda^{c}$; note also that $\exp \left\{-E^{\phi}(x, \gamma)\right\} \leq 1$; therefore, sum and integral in (3.3) are finite.

In [5], it was shown that the mapping $\hat{L}:=K^{-1} L K$ given on $B_{\mathrm{bs}}\left(\Gamma_{0}\right)$ by the following expression

$$
\begin{aligned}
(\hat{L} G)(\eta)= & -|\eta| G(\eta) \\
& +z \sum_{\xi \subset \eta} \int_{\mathbb{R}^{d}} e^{-E^{\phi}(x, \xi)} G(\xi \cup x) e_{\lambda}\left(e^{-\phi(x-\cdot)}-1, \eta \backslash \xi\right) d x
\end{aligned}
$$

is a linear operator on $\mathcal{L}_{C}$ with the dense domain $D(\hat{L})=\mathcal{L}_{2 C} \subset \mathcal{L}_{C}$. If, additionally,

$$
z \leq \min \left\{C e^{-C C_{\phi}} ; 2 C e^{-2 C C_{\phi}}\right\},
$$

then $(\hat{L}, D(\hat{L}))$ is closable linear operator in $\mathcal{L}_{C}$ and its closure (which we denote by $\hat{L}$ also) generates a strongly continuous contraction semigroup $\hat{T}(t)$ on $\mathcal{L}_{C}$.

Let us define $d \lambda_{C}:=C^{|\cdot|} d \lambda$. The topologically dual space is the space $\left(\mathcal{L}_{C}\right)^{\prime}=\left(L^{1}\left(\Gamma_{0}, d \lambda_{C}\right)\right)^{\prime}=L^{\infty}\left(\Gamma_{0}, d \lambda_{C}\right)$. The space $L^{\infty}\left(\Gamma_{0}, d \lambda_{C}\right)$ is isometrically isomorphic to the Banach space

$$
\mathcal{K}_{C}:=\left\{k: \Gamma_{0} \rightarrow \mathbb{R} \mid k \cdot C^{-|\cdot|} \in L^{\infty}\left(\Gamma_{0}, \lambda\right)\right\}
$$

with the norm $\|k\|_{\mathcal{K}_{C}}:=\left\|C^{-|\cdot|} k(\cdot)\right\|_{L^{\infty}\left(\Gamma_{0}, \lambda\right)}$, where the isomorphism is provided by the isometry $R_{C}$

$$
\left(\mathcal{L}_{C}\right)^{\prime} \ni k \longmapsto R_{C} k:=k \cdot C^{|\cdot|} \in \mathcal{K}_{C} .
$$

In fact, we may say about a duality between Banach spaces $\mathcal{L}_{C}$ and $\mathcal{K}_{C}$, which is given by the following expression

$$
\langle\langle G, k\rangle\rangle:=\int_{\Gamma_{0}} G \cdot k d \lambda, \quad G \in \mathcal{L}_{C}, k \in \mathcal{K}_{C}
$$


with

$$
|\langle\langle G, k\rangle\rangle| \leq\|G\|_{C} \cdot\|k\|_{\mathcal{K}_{C}} .
$$

It is clear that for any $k \in \mathcal{K}_{C}$

$$
|k(\eta)| \leq\|k\|_{\mathcal{K}_{C}} C^{|\eta|} \quad \text { for } \lambda \text {-a.a. } \eta \in \Gamma_{0} .
$$

Let $\left(\hat{L}^{\prime}, D\left(\hat{L}^{\prime}\right)\right)$ be an operator in $\left(\mathcal{L}_{C}\right)^{\prime}$ which is dual to the closed operator $(\hat{L}, D(\hat{L}))$. We consider also its image in $\mathcal{K}_{C}$ under isometry $R_{C}$, namely, let $\hat{L}^{*}=R_{C} \hat{L}^{\prime} R_{C^{-1}}$ with a domain $D\left(\hat{L}^{*}\right)=R_{C} D\left(\hat{L}^{\prime}\right)$. Then, for any $G \in D(\hat{L})$, $k \in D\left(\hat{L}^{*}\right)$

$$
\begin{aligned}
\int_{\Gamma_{0}} G \cdot \hat{L}^{*} k d \lambda & =\int_{\Gamma_{0}} G \cdot R_{C} \hat{L}^{\prime} R_{C^{-1}} k d \lambda=\int_{\Gamma_{0}} G \cdot \hat{L}^{\prime} R_{C^{-1}} k d \lambda_{C} \\
& =\int_{\Gamma_{0}} \hat{L} G \cdot R_{C^{-1}} k d \lambda_{C}=\int_{\Gamma_{0}} \hat{L} G \cdot k d \lambda
\end{aligned}
$$

therefore, $\hat{L}^{*}$ is the dual operator to $\hat{L}$ w.r.t. the duality (3.7).

By [7, we have the precise form of $\hat{L}^{*}$ on $D\left(\hat{L}^{*}\right)$ :

$$
\begin{aligned}
\left(\hat{L}^{*} k\right)(\eta)= & -|\eta| k(\eta) \\
& +z \sum_{x \in \eta} e^{-E^{\phi}(x, \eta \backslash x)} \int_{\Gamma_{0}} e_{\lambda}\left(e^{-\phi(x-\cdot)}-1, \xi\right) k((\eta \backslash x) \cup \xi) d \lambda(\xi) .
\end{aligned}
$$

Under condition (3.5), we consider the adjoint semigroup $\hat{T}^{\prime}(t)$ in $\left(\mathcal{L}_{C}\right)^{\prime}$ and its image $\hat{T}^{*}(t)$ in $\mathcal{K}_{C}$. Now, we may apply general results about adjoint semigroups (see, e.g., 3]) onto the semigroup $\hat{T}^{*}(t)$. The last semigroup will be weak ${ }^{*}$-continuous, moreover, weak*-differentiable at 0 and $\hat{L}^{*}$ will be weak*generator of $\hat{T}^{*}(t)$. Here and below we mean "weak*-properties" w.r.t. duality (3.7). Let

$$
\stackrel{\circ}{\mathcal{K}}_{C}=\left\{k \in \mathcal{K}_{C} \mid \exists \lim _{t \downarrow 0}\left\|\hat{T}^{*}(t) k-k\right\|_{\mathcal{K}_{C}}=0\right\} .
$$

Then $\mathcal{K}_{C}$ is a closed, weak ${ }^{*}$-dense, $\hat{T}^{*}(t)$-invariant linear subspace of $\mathcal{K}_{C}$. Moreover, $\dot{\mathcal{K}}_{C}=\overline{D\left(\hat{L}^{*}\right)}$ (the closure is in the norm of $\mathcal{K}_{C}$ ). Let $\hat{T}^{\odot}(t)$ denote the restriction of $\hat{T}^{*}(t)$ onto Banach space $\mathcal{K}_{C}$. Then $\hat{T}^{\odot}(t)$ is a $C_{0}$-semigroup on $\stackrel{\mathcal{K}}{C}_{C}$ and its generator $\hat{L}^{\odot}$ will be part of $\hat{L}^{*}$, namely,

$$
D\left(\hat{L}^{\odot}\right)=\left\{k \in D\left(\hat{L}^{*}\right) \mid \hat{L}^{*} k \in \overline{D\left(\hat{L}^{*}\right)}\right\}
$$

and $\hat{L}^{*} k=\hat{L}^{\odot} k$ for any $k \in D\left(\hat{L}^{\odot}\right)$.

And now we consider another $\hat{T}^{*}(t)$-invariant subspace. We present, at first, the useful subspace in $D\left(\hat{L}^{*}\right)$.

Proposition 3.1. For any $\alpha \in(0 ; 1)$ the following inclusions hold $\mathcal{K}_{\alpha C} \subset$ $D\left(\hat{L}^{*}\right) \subset \overline{D\left(\hat{L}^{*}\right)} \subset \mathcal{K}_{C}$. 
Proof. Let $\alpha \in(0 ; 1)$ and $k \in \mathcal{K}_{\alpha C}$ then, using (2.4) and (3.9), for $\lambda$-a.a. $\eta \in \Gamma_{0}$ we may estimate

$$
\begin{aligned}
& C^{-|\eta|}|\eta||k(\eta)|+\sum_{x \in \eta} \int_{\Gamma_{0}} e_{\lambda}\left(1-e^{-\phi(x-\cdot)}, \xi\right) C^{-|\eta|}|k((\eta \backslash x) \cup \xi)| d \lambda(\xi) \\
\leq & C^{-|\eta|}|\eta|\|k\|_{\mathcal{K}_{\alpha C}}(\alpha C)^{|\eta|} \\
& +\sum_{x \in \eta} \int_{\Gamma_{0}} e_{\lambda}\left(1-e^{-\phi(x-\cdot)}, \xi\right) C^{-|\eta|}\|k\|_{\mathcal{K}_{\alpha C}}(\alpha C)^{|(\eta \backslash x) \cup \xi|} d \lambda(\xi) \\
= & \alpha^{|\eta|}|\eta|\|k\|_{\mathcal{K}_{\alpha C}}+\frac{1}{\alpha C}\|k\|_{\mathcal{K}_{\alpha C}} \alpha^{|\eta|} \sum_{x \in \eta} \int_{\Gamma_{0}} e_{\lambda}\left(\alpha C\left(1-e^{-\phi(x-\cdot)}\right), \xi\right) d \lambda(\xi) \\
= & \alpha^{|\eta|}|\eta|\|k\|_{\mathcal{K}_{\alpha C}}+\frac{1}{\alpha C}\|k\|_{\mathcal{K}_{\alpha C}} \alpha^{|\eta|}|\eta| \exp \left\{\alpha C C_{\phi}\right\} \\
\leq & \|k\|_{\mathcal{K}_{\alpha C}} \frac{-1}{e \ln \alpha}\left(1+\frac{1}{\alpha C} \exp \left\{\alpha C C_{\phi}\right\}\right),
\end{aligned}
$$

since $x \alpha^{x} \leq-\frac{1}{e \ln \alpha}$ for any $\alpha \in(0 ; 1)$ and $x \geq 0$. Using the definition of $D\left(\hat{L}^{*}\right)$ and Lemma 2.1 we get immediately the statement of the proposition.

Remark 3.2. By the same arguments, the set of all functions $k \in \mathcal{K}_{C}$ such that

$$
|k(\eta)| \leq \mathrm{const} \cdot \frac{1}{|\eta|} C^{|\eta|}, \quad \eta \in \Gamma_{0} \backslash\{\emptyset\}
$$

is a subset of $D\left(\hat{L}^{*}\right)$. Due to the elementary inequality $\alpha^{x}<$ const $\cdot x^{-1}$ for any $\alpha \in(0 ; 1), x>0$, we have that this set contains $\mathcal{K}_{\alpha C}$. But the smaller set $\mathcal{K}_{\alpha C}$ is more useful for our calculations.

Proposition 3.3. Suppose that (3.5) is satisfied. Furthermore, we additionally assume that

$$
z<C e^{-C C_{\phi}}, \quad \text { if } \quad C C_{\phi} \leq \ln 2 .
$$

Then there exists $\alpha_{0}=\alpha_{0}(z, \phi, C) \in(0 ; 1)$ such that for any $\alpha \in\left(\alpha_{0} ; 1\right)$ the set $\mathcal{K}_{\alpha C}$ is the $\hat{T}^{*}(t)$-invariant linear subspace of $\mathcal{K}_{C}$.

Proof. Let us consider function $f(x):=x e^{-x}, x \geq 0$. It has the following properties: $f$ is increasing on $[0 ; 1]$ from 0 to $e^{-1}$ and it is asymptotically decreasing on $[1 ;+\infty)$ from $e^{-1}$ to $0 ; f(x)<f(2 x)$ for $x \in(0, \ln 2) ; x=\ln 2$ is the only non-zero solution to $f(x)=f(2 x)$.

By assumption (3.5),$z C_{\phi} \leq \min \left\{C C_{\phi} e^{-C C_{\phi}}, 2 C C_{\phi} e^{-2 C C_{\phi}}\right\}$. Therefore, if $C C_{\phi} e^{-C C_{\phi}} \neq 2 C C_{\phi} e^{-2 C C_{\phi}}$ then (3.5) with necessity implies

$$
z C_{\phi}<e^{-1} \text {. }
$$

This inequality remains also true if $C C_{\phi}=\ln 2$ because of (3.12). Under condition (3.13), the equation $f(x)=z C_{\phi}$ has exactly two roots, say, $0<x_{1}<1<$ $x_{2}<+\infty$. Then, (3.12) implies $x_{1}<C C_{\phi}<2 C C_{\phi} \leq x_{2}$. 
If $C C_{\phi}>1$ then we set $\alpha_{0}:=\max \left\{\frac{1}{2} ; \frac{1}{C C_{\phi}} ; \frac{1}{C}\right\}<1$. This yields $2 \alpha C C_{\phi}>C C_{\phi}$ and $\alpha C C_{\phi}>1>x_{1}$. If $x_{1}<C C_{\phi} \leq 1$ then we set $\alpha_{0}:=\max \left\{\frac{1}{2} ; \frac{x_{1}}{C C_{\phi}} ; \frac{1}{C}\right\}<1$ that gives $2 \alpha C C_{\phi}>C C_{\phi}$ and $\alpha C C_{\phi}>x_{1}$.

As a result,

$$
x_{1}<\alpha C C_{\phi}<C C_{\phi}<2 \alpha C C_{\phi}<2 C C_{\phi} \leq x_{2}
$$

and $1<\alpha C<C<2 \alpha C<2 C$. The last inequality shows that $\mathcal{L}_{2 C} \subset \mathcal{L}_{2 \alpha C} \subset$ $\mathcal{L}_{C} \subset \mathcal{L}_{\alpha C}$. Moreover, by (3.14), we may prove that the operator $\left(\hat{L}, \mathcal{L}_{2 \alpha C}\right)$ is closable in $\mathcal{L}_{\alpha C}$ and its closure is a generator of a contraction semigroup $\hat{T}_{\alpha}(t)$ on $\mathcal{L}_{\alpha C}$. The proof is identical to that in [5].

It is easy to see, that $\hat{T}_{\alpha}(t) G=\hat{T}(t) G$ for any $G \in \mathcal{L}_{C}$. Indeed, from the construction of the semigroup $\hat{T}(t)$, see [5], and analogous construction for the semigroup $\hat{T}_{\alpha}(t)$, we have that there exists family of mappings $\hat{P}_{\delta}, \delta>0$ independent of $\alpha$ and $C$, namely,

$$
\begin{aligned}
\left(\hat{P}_{\delta} G\right)(\eta):= & \sum_{\xi \subset \eta}(1-\delta)^{|\xi|} \int_{\Gamma_{0}}(z \delta)^{|\omega|} G(\xi \cup \omega) \\
& \times \prod_{y \in \xi} e^{-E^{\phi}(y, \omega)} \prod_{y \in \eta \backslash \xi}\left(e^{-E^{\phi}(y, \omega)}-1\right) d \lambda(\omega), \quad \eta \in \Gamma_{0} .
\end{aligned}
$$

such that $\hat{P}_{\delta}^{\left[\frac{t}{\delta}\right]}$ for any $t \geq 0$ strongly converges to $\hat{T}(t)$ and $\hat{T}_{\alpha}(t)$ in $\mathcal{L}_{C}$ and $\mathcal{L}_{\alpha C}$, correspondingly, as $\delta \rightarrow 0$. Here and below [.] means the entire part of a number. Then for any $G \in \mathcal{L}_{C} \subset \mathcal{L}_{\alpha C}$ we have that $\hat{T}(t) G \in \mathcal{L}_{C} \subset \mathcal{L}_{\alpha C}$ and $\hat{T}_{\alpha}(t) G \in \mathcal{L}_{\alpha C}$ and

$$
\begin{aligned}
\left\|\hat{T}(t) G-\hat{T}_{\alpha}(t) G\right\|_{\alpha C} & \leq\left\|\hat{T}(t) G-\hat{P}_{\delta}^{\left[\frac{t}{\delta}\right]} G\right\|_{\alpha C}+\left\|\hat{T}_{\alpha}(t) G-\hat{P}_{\delta}^{\left[\frac{t}{\delta}\right]} G\right\|_{\alpha C} \\
& \leq\left\|\hat{T}(t) G-\hat{P}_{\delta}^{\left[\frac{t}{\delta}\right]} G\right\|_{C}+\left\|\hat{T}_{\alpha}(t) G-\hat{P}_{\delta}^{\left[\frac{t}{\delta}\right]} G\right\|_{\alpha C} \rightarrow 0,
\end{aligned}
$$

as $\delta \rightarrow 0$. Therefore, $\hat{T}(t) G=\hat{T}_{\alpha}(t) G$ in $\mathcal{L}_{\alpha C}$ (recall that $G \in \mathcal{L}_{C}$ ) that yields $\hat{T}(t) G(\eta)=\hat{T}_{\alpha}(t) G(\eta)$ for $\lambda$-a.a. $\eta \in \Gamma_{0}$ and, therefore, $\hat{T}(t) G=\hat{T}_{\alpha}(t) G$ in $\mathcal{L}_{C}$.

Note that for any $G \in \mathcal{L}_{C} \subset \mathcal{L}_{\alpha C}$ and for any $k \in \mathcal{K}_{\alpha C} \subset \mathcal{K}_{C}$ we have $\hat{T}_{\alpha}(t) G \in \mathcal{L}_{\alpha C}$ and

$$
\left\langle\left\langle\hat{T}_{\alpha}(t) G, k\right\rangle\right\rangle=\left\langle\left\langle G, \hat{T}_{\alpha}^{*}(t) k\right\rangle\right\rangle,
$$

where, by construction, $\hat{T}_{\alpha}^{*}(t) k \in \mathcal{K}_{\alpha C}$. But $G \in \mathcal{L}_{C}, k \in \mathcal{K}_{C}$ implies

$$
\left\langle\left\langle\hat{T}_{\alpha}(t) G, k\right\rangle\right\rangle=\langle\langle\hat{T}(t) G, k\rangle\rangle=\left\langle\left\langle G, \hat{T}^{*}(t) k\right\rangle\right\rangle \text {. }
$$

Hence, $\hat{T}^{*}(t) k=\hat{T}_{\alpha}^{*}(t) k \in \mathcal{K}_{\alpha C}, k \in \mathcal{K}_{\alpha C}$ that proves the statement.

Remark 3.4. As a result, (3.5) implies that for any $k_{0} \in \overline{D\left(\hat{L}^{*}\right)}$ the Cauchy problem in $\mathcal{K}_{C}$

$$
\left\{\begin{array}{l}
\frac{\partial}{\partial t} k_{t}=\hat{L}^{*} k_{t} \\
\left.k_{t}\right|_{t=0}=k_{0}
\end{array}\right.
$$


has a unique mild solution: $k_{t}=\hat{T}^{*}(t) k_{0}=\hat{T}^{\odot}(t) k_{0} \in \overline{D\left(\hat{L}^{*}\right)}$. Moreover, $k_{0} \in \mathcal{K}_{\alpha C}$ implies $k_{t} \in \mathcal{K}_{\alpha C}$ provided (3.12) is satisfied.

Remark 3.5. The Cauchy problem (3.16) is well-posed in $\mathcal{K}_{C}=\overline{D\left(\hat{L}^{*}\right)}$, i.e., for every $k_{0} \in D\left(\hat{L}^{\odot}\right)$ there exists a unique solution $k_{t} \in \dot{\mathcal{K}}_{C}$ of (3.16).

Let (3.5) and (3.12) be satisfied and let $\alpha_{0}$ be chosen as in the proof of Proposition 3.3 and fixed. Suppose that $\alpha \in\left(\alpha_{0} ; 1\right)$. Then, Propositions 3.1 and 3.3 imply $\overline{\mathcal{K}_{\alpha C}} \subset \overline{D\left(\hat{L}^{*}\right)}$ and the Banach subspace $\overline{\mathcal{K}_{\alpha C}}$ is $\hat{T}^{*}(t)$ - and, therefore, $\hat{T}^{\odot}(t)$-invariant due to the continuity of these operators.

Let now $\hat{T}^{\odot \alpha}(t)$ be the restriction of the strongly continuous semigroup $\hat{T}^{\odot}(t)$ onto the closed linear subspace $\overline{\mathcal{K}_{\alpha C}}$. By general result (see, e.g., [3]), $\hat{T}^{\odot \alpha}(t)$ is a strongly continuous semigroups on $\overline{\mathcal{K}_{\alpha C}}$ with generator $\hat{L}^{\odot \alpha}$ which is the restriction of the operator $\hat{L} \odot$. Namely,

$$
D\left(\hat{L}^{\odot \alpha}\right)=\left\{k \in \overline{\mathcal{K}_{\alpha C}} \mid \hat{L}^{*} k \in \overline{\mathcal{K}_{\alpha C}}\right\}
$$

and

$$
\hat{L}^{\odot \alpha} k=\hat{L}^{\odot} k=\hat{L}^{*} k, \quad k \in D\left(\hat{L}^{\odot \alpha}\right)
$$

Since $\hat{T}(t)$ is a contraction semigroup on $\mathcal{L}_{C}$, then, $\hat{T}^{\prime}(t)$ is also a contraction semigroup on $\left(\mathcal{L}_{C}\right)^{\prime}$; but isomorphism (3.6) is isometrical, therefore, $\hat{T}^{*}(t)$ is a contraction semigroup on $\mathcal{K}_{C}$. As a result, its restriction $\hat{T}^{\odot \alpha}(t)$ is a contraction semigroup on $\overline{\mathcal{K}_{\alpha C}}$. Note also, that by (3.17),

$$
D_{\alpha C}:=\left\{\begin{array}{l|l}
k \in \mathcal{K}_{\alpha C} & \left.\hat{L}^{*} k \in \overline{\mathcal{K}_{\alpha C}}\right\}
\end{array}\right.
$$

is a core for $\hat{L}^{\odot \alpha}$ in $\overline{\mathcal{K}_{\alpha C}}$.

By (3.15), for any $k \in \mathcal{K}_{\alpha C}, G \in B_{\mathrm{bs}}\left(\Gamma_{0}\right)$ we have

$$
\begin{aligned}
& \int_{\Gamma_{0}}\left(\hat{P}_{\delta} G\right)(\eta) k(\eta) d \lambda(\eta) \\
= & \int_{\Gamma_{0}} \sum_{\xi \subset \eta}(1-\delta)^{|\xi|} \int_{\Gamma_{0}}(z \delta)^{|\omega|} G(\xi \cup \omega) \prod_{y \in \xi} e^{-E^{\phi}(y, \omega)} \\
& \times \prod_{y \in \eta \backslash \xi}\left(e^{-E^{\phi}(y, \omega)}-1\right) d \lambda(\omega) k(\eta) d \lambda(\eta) \\
= & \int_{\Gamma_{0}} \int_{\Gamma_{0}}(1-\delta)^{|\xi|} \int_{\Gamma_{0}}(z \delta)^{|\omega|} G(\xi \cup \omega) \prod_{y \in \xi} e^{-E^{\phi}(y, \omega)} \\
& \times \prod_{y \in \eta}\left(e^{-E^{\phi}(y, \omega)}-1\right) d \lambda(\omega) k(\eta \cup \xi) d \lambda(\xi) d \lambda(\eta) \\
= & \int_{\Gamma_{0}} \int_{\Gamma_{0}} \sum_{\omega \subset \xi}(1-\delta)^{|\xi \backslash \omega|}(z \delta)^{|\omega|} G(\xi) \prod_{y \in \xi \backslash \omega} e^{-E^{\phi}(y, \omega)} \\
& \times \prod_{y \in \eta}\left(e^{-E^{\phi}(y, \omega)}-1\right) k(\eta \cup \xi \backslash \omega) d \lambda(\xi) d \lambda(\eta),
\end{aligned}
$$


therefore,

$$
\begin{aligned}
\left(\hat{P}_{\delta}^{*} k\right)(\eta)= & \sum_{\omega \subset \eta}(1-\delta)^{|\eta \backslash \omega|}(z \delta)^{|\omega|} \prod_{y \in \eta \backslash \omega} e^{-E^{\phi}(y, \omega)} \\
& \times \int_{\Gamma_{0}} \prod_{y \in \xi}\left(e^{-E^{\phi}(y, \omega)}-1\right) k(\xi \cup \eta \backslash \omega) d \lambda(\xi) .
\end{aligned}
$$

Proposition 3.6. Suppose that (3.5) and (3.12) are fulfilled. Then, for any $k \in D_{\alpha C}$ and $\alpha \in\left(\alpha_{0}, 1\right)$, where $\alpha_{0}$ is chosen as in the proof of Proposition 3.3.

$$
\lim _{\delta \rightarrow 0}\left\|\frac{1}{\delta}\left(\hat{P}_{\delta}^{*}-\mathbb{1}\right) k-\hat{L}^{\odot \alpha} k\right\|_{\mathcal{K}_{C}}=0 .
$$

Proof. Let us recall (3.10) and define

$$
\begin{aligned}
\left(\hat{P}_{\delta}^{*,(0)} k\right)(\eta)= & (1-\delta)^{|\eta|} k(\eta) ; \\
\left(\hat{P}_{\delta}^{*,(1)} k\right)(\eta)= & z \delta \sum_{x \in \eta}(1-\delta)^{|\eta|-1} e_{\lambda}\left(e^{-\phi(x-\cdot)}, \eta \backslash x\right) \\
& \times \int_{\Gamma_{0}} e_{\lambda}\left(e^{-\phi(x-\cdot)}-1, \xi\right) k(\xi \cup \eta \backslash x) d \lambda(\xi) ;
\end{aligned}
$$

and $\hat{P}_{\delta}^{*,(\geq 2)}=\hat{P}_{\delta}^{*}-\hat{P}_{\delta}^{*,(0)}-\hat{P}_{\delta}^{*,(1)}$.

We will use the following elementary inequality, for any $n \in \mathbb{N} \cup\{0\}, \delta \in(0 ; 1)$

$$
0 \leq n-\frac{1-(1-\delta)^{n}}{\delta} \leq \delta \frac{n(n-1)}{2} .
$$

Then, for any $k \in \mathcal{K}_{\alpha C}$ and $\lambda$-a.a. $\eta \in \Gamma_{0}, \eta \neq \emptyset$

$$
\begin{aligned}
& C^{-|\eta|}\left|\frac{1}{\delta}\left(\hat{P}_{\delta, \varepsilon}^{*,(0)}-\mathbb{1}\right) k(\eta)+\right| \eta|k(\eta)| \\
\leq & \|k\|_{\mathcal{K}_{\alpha C}} \alpha^{|\eta|}|| \eta\left|-\frac{1-(1-\delta)^{|\eta|}}{\delta}\right| \leq \frac{\delta}{2}\|k\|_{\mathcal{K}_{\alpha C}} \alpha^{|\eta|}|\eta|(|\eta|-1)
\end{aligned}
$$

and the function $\alpha^{x} x(x-1)$ is bounded for $x \geq 1, \alpha \in(0 ; 1)$. Next, for any $k \in \mathcal{K}_{\alpha C}$ and $\lambda$-a.a. $\eta \in \Gamma_{0}, \eta \neq \emptyset$

$$
\begin{aligned}
& \quad C^{-|\eta|} \mid \frac{1}{\delta} \hat{P}_{\delta}^{*,(1)} k(\eta)-z \sum_{x \in \eta} \int_{\Gamma_{0}} e_{\lambda}\left(e^{-\phi(x-\cdot)}, \eta \backslash x\right) \\
& \quad \times e_{\lambda}\left(e^{-\phi(x-\cdot)}-1, \xi\right) k(\xi \cup \eta \backslash x) d \lambda(\xi) \mid \\
& \leq\|k\|_{\mathcal{K}_{\alpha C}} \frac{z}{\alpha C} \alpha^{|\eta|} \sum_{x \in \eta}\left(1-(1-\delta)^{|\eta|-1}\right) \int_{\Gamma_{0}} e_{\lambda}\left(\alpha C\left(e^{-\phi(x-\cdot)}-1\right), \xi\right) d \lambda(\xi) \\
& \leq\|k\|_{\mathcal{K}_{\alpha C}} \frac{z}{\alpha C} \alpha^{|\eta|} \sum_{x \in \eta}\left(1-(1-\delta)^{|\eta|-1}\right) \exp \left\{\alpha C C_{\phi}\right\} \\
& \leq\|k\|_{\mathcal{K}_{\alpha C}} \frac{z}{\alpha C} \alpha^{|\eta|} \delta|\eta|(|\eta|-1) \exp \left\{\alpha C C_{\phi}\right\} .
\end{aligned}
$$


which is small in $\delta$ uniformly by $|\eta|$. Now, using inequality

$$
1-e^{-E^{\phi}(y, \omega)}=1-\prod_{x \in \omega} e^{-\phi(x-y)} \leq \sum_{x \in \omega}\left(1-e^{-\phi(x-y)}\right),
$$

we obtain

$$
\begin{array}{r}
\frac{1}{\delta} C^{-|\eta|} \sum_{\substack{\omega \subset \eta \\
|\omega| \geq 2}}(1-\delta)^{|\eta \backslash \omega|}(z \delta)^{|\omega|} e_{\lambda}\left(e^{-E^{\phi}(\cdot, \omega)}, \eta \backslash \omega\right) \\
\times \int_{\Gamma_{0}} e_{\lambda}\left(\left|e^{-E^{\phi}(\cdot, \omega)}-1\right|, \xi\right)|k(\xi \cup \eta \backslash \omega)| d \lambda(\xi) \\
=\|k\|_{\mathcal{K}_{\alpha C}} \alpha^{|\eta|} \frac{1}{\delta} \sum_{\substack{\omega \subset \eta \\
|\omega| \geq 2}}(1-\delta)^{|\eta \backslash \omega|}\left(\frac{z \delta}{\alpha C} \exp \left\{\alpha C C_{\phi}\right\}\right)^{|\omega|} ;
\end{array}
$$

recall that $\alpha>\alpha_{0}$, therefore, $z \exp \left\{\alpha C C_{\phi}\right\} \leq \alpha C$, and one may continue

$$
\begin{aligned}
& \leq\|k\|_{\mathcal{K}_{\alpha C}} \alpha^{|\eta|} \frac{1}{\delta} \sum_{\substack{\omega \subset \eta \\
|\omega| \geq 2}}(1-\delta)^{|\eta| \omega \mid} \delta^{|\omega|} \\
& =\|k\|_{\mathcal{K}_{\alpha C} \delta} \delta \alpha^{|\eta|} \sum_{k=2}^{|\eta|} \frac{|\eta| !}{k !(|\eta|-k) !}(1-\delta)^{|\eta|-k} \delta^{k-2} \\
& =\|k\|_{\mathcal{K}_{\alpha C} \delta} \delta \alpha^{|\eta|} \sum_{k=0}^{|\eta|-2} \frac{|\eta| !}{(k+2) !(|\eta|-k-2) !}(1-\delta)^{|\eta|-k-2} \delta^{k} \\
& =\|k\|_{\mathcal{K}_{\alpha C} \delta} \delta \alpha^{|\eta|}|\eta|(|\eta|-1) \sum_{k=0}^{|\eta|-2} \frac{(|\eta|-2) !}{(k+2) !(|\eta|-k-2) !}(1-\delta)^{|\eta|-2-k} \delta^{k} \\
& \leq\|k\|_{\mathcal{K}_{\alpha C} \delta} \delta \alpha^{|\eta|}|\eta|(|\eta|-1) \sum_{k=0}^{|\eta|-2} \frac{(|\eta|-2) !}{k !(|\eta|-k-2) !}(1-\delta)^{|\eta|-2-k} \delta^{k} \\
& =\|k\|_{\mathcal{K}_{\alpha C} \delta} \delta \alpha^{|\eta|}|\eta|(|\eta|-1) .
\end{aligned}
$$

Combining inequalities (3.21)-(3.23) we obtain (3.20).

We recall now well-known approximation result (cf., e.g., [4, Theorem 6.5])

Lemma 3.7. Let $L, L_{n}, n \in \mathbb{N}$ be Banach spaces, and $p_{n}: L \rightarrow L_{n}$ be bounded linear transformation, such that $\sup _{n}\left\|p_{n}\right\|<\infty$. For any $n \in \mathbb{N}$, let $T_{n}$ be a linear contraction on $L_{n}$, let $\varepsilon_{n}>0$ be such that $\lim _{n \rightarrow \infty} \varepsilon_{n}=0$, and put $A_{n}=\varepsilon_{n}^{-1}\left(T_{n}-\mathbb{1}\right)$. Let $T_{t}$ be a strongly continuous contraction semigroup on $L$ with generator $A$ and let $D$ be a core for $A$. Then the following are equivalent:

1. For each $f \in L,\left\|T_{n}^{\left[t / \varepsilon_{n}\right]} p_{n} f-p_{n} T_{t} f\right\|_{L_{n}} \rightarrow 0, n \rightarrow \infty$ for all $t \geq 0$ uniformly on bounded intervals. Here and below [.] mean the entire part of a real number. 
2. For each $f \in D$, there exists $f_{n} \in L_{n}$ for each $n \in \mathbb{N}$ such that $\left\|f_{n}-p_{n} f\right\|_{L_{n}} \rightarrow 0$ and $\left\|A_{n} f_{n}-p_{n} A f\right\|_{L_{n}} \rightarrow 0, n \rightarrow \infty$.

Theorem 3.8. Let $\alpha_{0}$ be chosen as in the proof of the Proposition 3.3 and be fixed. Let $\alpha \in\left(\alpha_{0} ; 1\right)$ and $k \in \overline{\mathcal{K}_{\alpha C}}$ be given. Then

$$
\left(\hat{P}_{\delta}^{*}\right)^{[t / \delta]} k \rightarrow \hat{T}^{\odot \alpha}(t) k, \quad \delta \rightarrow 0
$$

in the space $\overline{\mathcal{K}_{\alpha C}}$ with norm $\|\cdot\|_{\mathcal{K}_{C}}$ for all $t \geq 0$ uniformly on bounded intervals. Proof. We may apply Proposition 3.6 to use Lemma 3.7 in the case $L_{n}=L=$ $\overline{\mathcal{L}_{\alpha C}}, p_{n}=\mathbb{1}, f_{n}=f=k, \varepsilon_{n}=\delta \rightarrow 0, n \in \mathbb{N}$.

\section{Positive definiteness}

Definition 4.1. A measurable function $k: \Gamma_{0} \rightarrow \mathbb{R}$ is called a positive defined function (cf. [16, 17]) if for any $G \in L_{\mathrm{ls}}^{0}\left(\Gamma_{0}\right)$ such that $K G \geq 0$ and $G \in \mathcal{L}_{C}$ for some $C>1$ the following inequality holds

$$
\int_{\Gamma_{0}} G(\eta) k(\eta) d \lambda(\eta) \geq 0
$$

In [16, 17], it was shown that if $k$ is a positive defined function and $|k(\eta)| \leq$ $C^{|\eta|}(|\eta| !)^{2}, \eta \in \Gamma_{0}$ then there exists a unique measure $\mu \in \mathcal{M}_{\mathrm{fm}}^{1}(\Gamma)$ such that $k=k_{\mu}$ be its correlation functional in the sense of (2.8). Our aim is to show that the evolution $k \mapsto \hat{T}_{t}^{\odot} k$ preserves the property of the positive definiteness.

Theorem 4.2. Let (3.5) holds and $k \in \overline{D\left(\hat{L}^{*}\right)} \subset \mathcal{K}_{C}$ be a positive defined function. Then $k_{t}:=\hat{T}_{t}^{\odot} k \in \overline{D\left(\hat{L}^{*}\right)} \subset \mathcal{K}_{C}$ will be a positive defined function for any $t \geq 0$.

Proof. Let $C>0$ be arbitrary and fixed. For any $G \in \mathcal{L}_{C}^{\text {ls }}$ we have

$$
\int_{\Gamma_{0}} G(\eta) k_{t}(\eta) d \lambda(\eta)=\int_{\Gamma_{0}}\left(\hat{T}_{t} G\right)(\eta) k(\eta) d \lambda(\eta) .
$$

By [5, Proposition 3.10], under condition (3.5), we obtain that

$$
\lim _{n \rightarrow 0} \int_{\Gamma_{\Lambda_{n}}}\left|T_{n}^{[n t]} \mathbb{1}_{\Gamma_{\Lambda_{n}}} G(\eta)-\mathbb{1}_{\Gamma_{\Lambda_{n}}}(\eta)\left(\hat{T}_{t} G\right)(\eta)\right| C^{|\eta|} d \lambda(\eta)=0,
$$

where for $n \geq 2$

$$
T_{n}=\hat{P}_{\frac{1}{n}}^{\Lambda_{n}}
$$

and $\Lambda_{n} \nearrow \mathbb{R}^{d}$. Note that, by the dominated convergence theorem,

$$
\begin{aligned}
\int_{\Gamma_{0}}\left(\hat{T}_{t} G\right)(\eta) k(\eta) d \lambda(\eta) & =\lim _{n \rightarrow \infty} \int_{\Gamma_{0}} \mathbb{1}_{\Gamma_{\Lambda_{n}}}(\eta)\left(\hat{T}_{t} G\right)(\eta) k(\eta) d \lambda(\eta) \\
& =\lim _{n \rightarrow \infty} \int_{\Gamma_{\Lambda_{n}}}\left(\hat{T}_{t} G\right)(\eta) k(\eta) d \lambda(\eta)
\end{aligned}
$$


Next,

$$
\begin{aligned}
& \left|\int_{\Gamma_{\Lambda_{n}}}\left(\hat{T}_{t} G\right)(\eta) k(\eta) d \lambda(\eta)-\int_{\Gamma_{\Lambda_{n}}} T_{n}^{[n t]} \mathbb{1}_{\Gamma_{\Lambda_{n}}} G(\eta) k(\eta) d \lambda(\eta)\right| \\
\leq & \int_{\Gamma_{\Lambda_{n}}}\left|T_{n}^{[n t]} \mathbb{1}_{\Gamma_{\Lambda_{n}}} G(\eta)-\mathbb{1}_{\Gamma_{\Lambda_{n}}}(\eta)\left(\hat{T}_{t} G\right)(\eta)\right| k(\eta) d \lambda(\eta) \\
\leq & \|k\|_{\mathcal{K}_{C}} \int_{\Gamma_{\Lambda_{n}}}\left|T_{n}^{[n t]} \mathbb{1}_{\Gamma_{\Lambda_{n}}} G(\eta)-\mathbb{1}_{\Gamma_{\Lambda_{n}}}(\eta)\left(\hat{T}_{t} G\right)(\eta)\right| C^{|\eta|} d \lambda(\eta) \rightarrow 0, \quad n \rightarrow \infty .
\end{aligned}
$$

Therefore,

$$
\int_{\Gamma_{0}}\left(\hat{T}_{t} G\right)(\eta) k(\eta) d \lambda(\eta)=\lim _{n \rightarrow \infty} \int_{\Gamma_{\Lambda_{n}}} T_{n}^{[n t]} \mathbb{1}_{\Gamma_{\Lambda_{n}}} G(\eta) k(\eta) d \lambda(\eta) .
$$

Our aim is to show that for any $G \in \mathcal{L}_{C}^{\text {ls }}$ the inequality $K G \geq 0$ implies

$$
\int_{\Gamma_{0}} G(\eta) k_{t}(\eta) d \lambda(\eta) \geq 0
$$

By (4.1) and (4.2), it is enough to show that for any $m \in \mathbb{N}$ and for any $G \in \mathcal{L}_{C}^{\mathrm{ls}}$ such that $K G \geq 0$ the following inequality holds

$$
\int_{\Gamma_{0}} \mathbb{1}_{\Gamma_{\Lambda_{n}}} T_{n}^{m} \mathbb{1}_{\Gamma_{\Lambda_{n}}} G(\eta) k(\eta) d \lambda(\eta) \geq 0, \quad m \in \mathbb{N}_{0} .
$$

The inequality (4.3) is fulfilled if only

$$
K \mathbb{1}_{\Gamma_{\Lambda_{n}}} T_{n}^{m} G_{n} \geq 0
$$

where $G_{n}:=\mathbb{1}_{\Gamma_{\Lambda_{n}}} G$. Note that

$$
\begin{aligned}
\left(K \mathbb{1}_{\Gamma_{\Lambda_{n}}} T_{n}^{m} G_{n}\right)(\gamma) & =\sum_{\eta \Subset \gamma} \mathbb{1}_{\Gamma_{\Lambda_{n}}}(\eta)\left(T_{n}^{m} G_{n}\right)(\eta) \\
& =\sum_{\eta \subset \gamma_{\Lambda_{n}}}\left(T_{n}^{m} G_{n}\right)(\eta)=\left(K T_{n}^{m} G_{n}\right)\left(\gamma_{\Lambda_{n}}\right)
\end{aligned}
$$

for any $m \in \mathbb{N}_{0}$. In particular,

$$
\left(K G_{n}\right)(\gamma)=\left(K \mathbb{1}_{\Gamma_{\Lambda_{n}}} G\right)(\gamma)=(K G)\left(\gamma_{\Lambda_{n}}\right) \geq 0 .
$$

Let us now consider any $\tilde{G} \in \mathcal{L}_{C}^{\text {ls }}$ (stress that $\tilde{G}$ is not necessary equal to 0 outside of $\left.\Gamma_{\Lambda_{n}}\right)$ and suppose that $(K \tilde{G})(\gamma) \geq 0$ for any $\gamma \in \Gamma_{\Lambda_{n}}$. Then

$$
\begin{aligned}
& \left(K T_{n} \tilde{G}\right)\left(\gamma_{\Lambda_{n}}\right)=\left(K \hat{P}_{\frac{1}{n}}^{\Lambda_{n}} \tilde{G}\right)\left(\gamma_{\Lambda_{n}}\right)=\left(P_{\frac{1}{n}}^{\Lambda_{n}} K \tilde{G}\right)\left(\gamma_{\Lambda_{n}}\right) \\
= & \left(\Xi_{\frac{1}{n}}^{\Lambda_{n}}\left(\gamma_{\Lambda_{n}}\right)\right)^{-1} \sum_{\eta \subset \gamma_{\Lambda_{n}}}\left(\frac{1}{n}\right)^{|\eta|}\left(1-\frac{1}{n}\right)^{|\gamma \backslash \eta|} \\
& \times \int_{\Gamma_{\Lambda_{n}}}\left(\frac{z}{n}\right)^{|\omega|} \prod_{y \in \omega} e^{-E^{\phi}(y, \gamma)}(K \tilde{G})\left(\left(\gamma_{\Lambda_{n}} \backslash \eta\right) \cup \omega\right) d \lambda(\omega) \geq 0 .
\end{aligned}
$$


By (4.6), setting $\tilde{G}=G_{n} \in \mathcal{L}_{C}^{\text {ls }}$ we obtain, because of (4.7), $K T_{n} G_{n} \geq 0$. Next, setting $\tilde{G}=T_{n} G_{n} \in \mathcal{L}_{C}^{\text {ls }}$ we obtain, by (4.7), $K T_{n}^{2} G_{n} \geq 0$. Then, using an induction mechanism, we obtain that

$$
\left(K T_{n}^{m} G_{n}\right)\left(\gamma_{\Lambda_{n}}\right) \geq 0, \quad m \in \mathbb{N}_{0},
$$

that, by (4.4) and (4.5), yields (4.3). This completes the proof.

\section{Ergodicity}

Let $k \in \overline{\mathcal{K}_{\alpha C}}$ be such that $k(\emptyset)=0$ then, by (3.19),$\left(\hat{P}_{\delta}^{*} k\right)(\emptyset)=0$. Class of all such functions we denote by $\mathcal{K}_{\alpha}^{0}$.

Proposition 5.1. Assume that there exists $\nu \in(0 ; 1)$ such that

$$
z \leq \min \left\{\nu C e^{-C C_{\phi}} ; 2 C e^{-2 C C_{\phi}}\right\} \text {. }
$$

Let, additionally, $\alpha \in\left(\alpha_{0} ; 1\right)$, where $\alpha_{0}$ is chosen as in the proof of the Proposition 3.3. Then for any $\delta \in(0 ; 1)$ the following estimate holds

$$
\| \hat{P}_{\delta}^{*}\left\lceil\mathcal{K}_{\alpha}^{0} \| \leq 1-(1-\nu) \delta .\right.
$$

Proof. It is easily seen that for any $k \in \mathcal{K}_{\alpha}^{0}$ the following inequality holds

$$
|k(\eta)| \leq 1_{|\eta|>0}\|k\|_{\mathcal{K}_{C}} C^{|\eta|}, \quad \lambda \text {-a.a. } \eta \in \Gamma_{0} .
$$

Then, using (3.19), we have

$$
\begin{aligned}
& C^{-|\eta|}\left|\left(\hat{P}_{\delta}^{*} k\right)(\eta)\right| \\
& \leq C^{-|\eta|} \sum_{\omega \subset \eta}(1-\delta)^{|\eta \backslash \omega|}(z \delta)^{|\omega|} \int_{\Gamma_{0}} \prod_{y \in \xi}\left(1-e^{-E^{\phi}(y, \omega)}\right)|k(\xi \cup \eta \backslash \omega)| d \lambda(\xi) \\
& \leq\|k\|_{\mathcal{K}_{C}} \sum_{\omega \subset \eta}(1-\delta)^{|\eta \backslash \omega|}\left(\frac{z \delta}{C}\right)^{|\omega|} \int_{\Gamma_{0}} \prod_{y \in \xi}\left(1-e^{-E^{\phi}(y, \omega)}\right) C^{|\xi|} \mathbb{1}_{|\xi|+|\eta \backslash \omega|>0} d \lambda(\xi) \\
&=\|k\|_{\mathcal{K}_{C}} \sum_{\omega \subsetneq \eta}(1-\delta)^{|\eta \backslash \omega|}\left(\frac{z \delta}{C}\right)^{|\omega|} \int_{\Gamma_{0}} \prod_{y \in \xi}\left(1-e^{-E^{\phi}(y, \omega)}\right) C^{|\xi|} d \lambda(\xi) \\
&+\|k\|_{\mathcal{K}_{C}}\left(\frac{z \delta}{C}\right)^{|\eta|} \int_{\Gamma_{0}} \prod_{y \in \xi}\left(1-e^{-E^{\phi}(y, \omega)}\right) C^{|\xi|} \mathbb{1}_{|\xi|>0} d \lambda(\xi) \\
&=\|k\|_{\mathcal{K}_{C}} \sum_{\omega \subsetneq \eta}(1-\delta)^{|\eta \backslash \omega|}\left(\frac{z \delta}{C}\right)^{|\omega|} \int_{\Gamma_{0}} \prod_{y \in \xi}\left(1-e^{-E^{\phi}(y, \omega)}\right) C^{|\xi|} d \lambda(\xi) \\
&+\|k\|_{\mathcal{K}_{C}}\left(\frac{z \delta}{C}\right)^{|\eta|} \int_{\Gamma_{0}} \prod_{y \in \xi}\left(1-e^{-E^{\phi}(y, \omega)}\right) C^{|\xi|} d \lambda(\xi)-\|k\|_{\mathcal{K}_{C}}\left(\frac{z \delta}{C}\right)^{|\eta|}
\end{aligned}
$$




$$
\begin{aligned}
& =\|k\|_{\mathcal{K}_{C}} \sum_{\omega \subset \eta}(1-\delta)^{|\eta \backslash \omega|}\left(\frac{z \delta}{C}\right)^{|\omega|} \int_{\Gamma_{0}} \prod_{y \in \xi}\left(1-e^{-E^{\phi}(y, \omega)}\right) C^{|\xi|} d \lambda(\xi) \\
& -\|k\|_{\mathcal{K}_{C}}\left(\frac{z \delta}{C}\right)^{|\eta|} \\
& =\|k\|_{\mathcal{K}_{C}} \sum_{\omega \subset \eta}(1-\delta)^{|\eta \backslash \omega|}\left(\frac{z \delta}{C}\right)^{|\omega|} \exp \left\{C \int_{\mathbb{R}^{d}}\left(1-e^{-E^{\phi}(y, \omega)}\right) d y\right\} \\
& -\|k\|_{\mathcal{K}_{C}}\left(\frac{z \delta}{C}\right)^{|\eta|} \\
& \leq\|k\|_{\mathcal{K}_{C}} \sum_{\omega \subset \eta}(1-\delta)^{|\eta \backslash \omega|}\left(\frac{z \delta}{C}\right)^{|\omega|} \exp \left\{C C_{\beta}|\omega|\right\}-\|k\|_{\mathcal{K}_{C}}\left(\frac{z \delta}{C}\right)^{|\eta|} \\
& \leq\|k\|_{\mathcal{K}_{C}} \sum_{\omega \subset \eta}(1-\delta)^{|\eta \backslash \omega|}(\nu \delta)^{|\omega|}-\|k\|_{\mathcal{K}_{C}}\left(\frac{z \delta}{C}\right)^{|\eta|} \\
& =\|k\|_{\mathcal{K}_{C}}\left((1-(1-\nu) \delta)^{|\eta|}-\left(\frac{z \delta}{C}\right)^{|\eta|}\right) \\
& =\|k\|_{\mathcal{K}_{C}}\left(1-(1-\nu) \delta-\frac{z \delta}{C}\right) \sum_{j=0}^{|\eta|-1}(1-(1-\nu) \delta)^{|\eta|-1-|j|}\left(\frac{z \delta}{C}\right)^{j} \\
& \leq\|k\|_{\mathcal{K}_{C}}\left(1-(1-\nu) \delta-\frac{z \delta}{C}\right) \sum_{j=0}^{|\eta|-1}\left(\frac{z \delta}{C}\right)^{j} \\
& =\|k\|_{\mathcal{K}_{C}}\left(1-(1-\nu) \delta-\frac{z \delta}{C}\right) \frac{1-\left(\frac{z \delta}{C}\right)^{|\eta|}}{1-\frac{z \delta}{C}} \\
& \leq\|k\|_{\mathcal{K}_{C}}\left(1-(1-\nu) \delta-\frac{z \delta}{C}\right) \frac{1}{1-\frac{z \delta}{C}} \\
& =\|k\|_{\mathcal{K}_{C}}\left(1-\frac{(1-\nu) \delta}{1-\frac{z \delta}{C}}\right) \leq\|k\|_{\mathcal{K}_{C}}(1-(1-\nu) \delta),
\end{aligned}
$$

where we have used that, clearly, $z<\nu C<C$. The statement is proved.

Remark 5.2. Condition (5.1) is equivalent to (3.5) and (3.12).

Suppose that (cf. (3.13))

$$
z C_{\phi}<(2 e)^{-1},
$$

then (see, e.g., 6 for details) there exists a Gibbs measure $\mu$ on $(\Gamma, \mathcal{B}(\Gamma))$ corresponding to the potential $\phi \geq 0$ and activity parameter $z$. We denote the corresponding correlation function by $k_{\mu}$. The measure $\mu$ is reversible (symmetrizing) for the operator defined by (3.3) (see, e.g., [6], [13]). Therefore, for 
any $F \in K B_{\mathrm{bs}}\left(\Gamma_{0}\right)$

$$
\int_{\Gamma} L F(\gamma) d \mu(\gamma)=0 .
$$

Theorem 5.3. Let (5.3) and (5.1) hold and let $\alpha \in\left(\alpha_{0} ; 1\right)$, where $\alpha_{0}$ is chosen as in the proof of Proposition 3.3. Let $k_{0} \in \overline{\mathcal{K}_{\alpha C}}, k_{t}=\hat{T}^{\odot \alpha}(t) k_{0}$. Then for any $t \geq 0$

$$
\left\|k_{t}-k_{\mu}\right\|_{\mathcal{K}_{C}} \leq e^{-(1-\nu) t}\left\|k_{0}-k_{\mu}\right\|_{\mathcal{K}_{C}} .
$$

Proof. First of all, let us note that for any $\alpha \in\left(\alpha_{0} ; 1\right)$ the inequality (3.14) implies $z \leq \alpha C \exp \left\{-\alpha C C_{\phi}\right\}$. Hence $k_{\mu}(\eta) \leq(\alpha C)^{|\eta|}, \eta \in \Gamma_{0}$. Therefore, $k_{\mu} \in \mathcal{K}_{\alpha C} \subset \overline{\mathcal{K}_{\alpha C}} \cap D\left(\hat{L}^{*}\right)$. By (5.4), for any $G \in B_{\mathrm{bs}}\left(\Gamma_{0}\right)$ we have $\left\langle\left\langle\hat{L} G, k_{\mu}\right\rangle\right\rangle=0$. It means that $\hat{L}^{*} k_{\mu}=0$. Therefore, $\hat{L}^{\odot \alpha} k_{\mu}=0$. As a result, $\hat{T}^{\odot \alpha}(t) k_{\mu}=k_{\mu}$. Let $r_{0}=k_{0}-k_{\mu} \in \overline{\mathcal{K}_{\alpha C}}$. Then $r_{0} \in \mathcal{K}_{a}^{0}$ and

$$
\begin{aligned}
& \left\|k_{t}-k_{\mu}\right\|_{\mathcal{K}_{C}}=\left\|\hat{T}^{\odot \alpha}(t) r_{0}\right\|_{\mathcal{K}_{C}} \\
& \leq\left\|\left(\hat{P}_{\delta}^{*}\right)^{\left[\frac{t}{\delta}\right]} r_{0}\right\|_{\mathcal{K}_{C}}+\left\|\hat{T}^{\odot \alpha}(t) r_{0}-\left(\hat{P}_{\delta}^{*}\right)^{\left[\frac{t}{\delta}\right]} r_{0}\right\|_{\mathcal{K}_{C}} \\
& \leq \| \hat{P}_{\delta}^{*}\left\lceil\mathcal{K}_{\alpha}^{0}\left\|^{\left[\frac{t}{\delta}\right]} \cdot\right\| r_{0}\|\|_{\mathcal{K}_{C}}+\left\|\hat{T}^{\odot \alpha}(t) r_{0}-\left(\hat{P}_{\delta}^{*}\right)^{\left[\frac{t}{\delta}\right]} r_{0}\right\|_{\mathcal{K}_{C}}\right. \\
& \leq(1-(1-\nu) \delta)^{\frac{t}{\delta}-1}\left\|r_{0}\right\|_{\mathcal{K}_{C}}+\left\|\hat{T}^{\odot \alpha}(t) r_{0}-\left(\hat{P}_{\delta}^{*}\right)^{\left[\frac{t}{\delta}\right]} r_{0}\right\|_{\mathcal{K}_{C}},
\end{aligned}
$$

since $0<1-(1-\nu) \delta<1$ and $\frac{t}{\delta}<\left[\frac{t}{\delta}\right]+1$. Taking the limit as $\delta \downarrow 0$ in the right hand side of this inequality we obtain (5.5).

Acknowledgement The financial support of DFG through the SFB 701 (Bielefeld University) and German-Ukrainian Project 436 UKR 113/80 and 436 UKR $113 / 94$ is gratefully acknowledged. This work was partially supported by the Marie Curie "Transfer of Knowledge" program, project TODEQ (Warsaw, IMPAN). O.K. is very thankful to Prof. J. Zemanek for fruitful and stimulating discussions.

\section{References}

[1] Ph. Clèment, H. J. A. M. Heijmans, S. Angenent, C. J. van Duijn, and B. de Pagter, One-Parameter Semigroups, CWI Monographs 5, North-Holland, Amsterdam, 1987.

[2] R. L. Dobrushin, Ya. G. Sinai, and Yu. M. Sukhov, Dynamical Systems of Statistical Mechanics, in: Ergodic Theory with Applications to Dynamical Systems and Statistical Mechanics, Encyclopaedia Math. Sci., Vol. II, Springer, 1989.

[3] K.-J. Engel, R. Nagel, One-parameter Semigroups For Linear Evolution Equations, Springer, 2000. 
[4] S. N. Ethier, T. G. Kurtz, Markov Processes: Characterization and Convergence, Wiley \& Sons, 2005.

[5] D. L. Finkelshtein, Yu. G. Kondratiev, O. V. Kutoviy, and E. Zhizhina, An approximative approach to construction of the Glauber dynamics in continuum, CRC-701 Preprint 10041, University of Bielefeld, 2010.

[6] D. L. Finkelshtein, Yu. G. Kondratiev, and E. Lytvynov, Equilibrium Glauber dynamics of continuous particle systems as a scaling limit of Kawasaki dynamics, Random Oper. Stochastic Equations, 15 (2007), pp. 105-126.

[7] D. L. Finkelshtein, Yu. G. Kondratiev, and M. J. Oliveira,, Markov evolutions and hierarchical equations in the continuum I. One-component systems, J. Evol. Equ., 9 (2009), no. 2, pp. 197-233.

[8] N. L. Garcia, T. G. Kurtz, Spatial birth and death processes as solutions of stochastic equations. Alea. 1, 281-303 (2006)

[9] Yu. G. Kondratiev and T. Kuna, Harmonic analysis on configuration space. I. General theory, Infin. Dimens. Anal. Quantum Probab. Relat. Top., 5, no.2 (2002), pp. 201-233.

[10] Yu. G. Kondratiev and O. V. Kutoviy, On the metrical properties of the configuration space, Math. Nachr., 279, no.7 (2006), pp. 774-783.

[11] Yu. G. Kondratiev, O. V. Kutoviy, and R. A. Minlos, On non-equilibrium stochastic dynamics for interacting particle systems in continuum, J. Funct. Anal., 255 (2008), no. 1, pp. 200-227.

[12] Yu. G. Kondratiev, O. V. Kutoviy, and E. Zhizhina, Nonequilibrium Glauber-type dynamics in continuum, J. Math. Phys. 47 no. 11 (2006) 17 pp.

[13] Yu. G. Kondratiev and E. Lytvynov, Glauber dynamics of continuous particle systems, Ann. Inst. H.Poincare, Ser. A, Probab. Statist. 41 (2005), pp. 685-702.

[14] Yu. G. Kondratiev, E. Lytvynov, and M. Röckner, Equilibrium Kawasaki dynamics of continuous particle systems, Infin. Dimens. Anal. Quantum Probab. Relat. Top. 10 (2007), pp. 185-210.

[15] Yu. G. Kondratiev, R. Minlos, and E. Zhizhina, One-particle subspaces of the generator of Glauber dynamics of continuous particle systems, Rev. Math. Phys., 16, no. 9 (2004), pp. 1-42.

[16] A. Lenard, States of classical statistical mechanical systems of infinitely many particles. I, Arch. Rational Mech. Anal., 59 (1975), pp. 219-239.

[17] A. Lenard, States of classical statistical mechanical systems of infinitely many particles. II, Arch. Rational Mech. Anal., 59 (1975), pp. 241-256. 
[18] J. van Neerven, The Adjoint of a Semigroup of Linear Operators, SpringerVerlag, 1992.

[19] D. Ruelle, Statistical Mechanics, New York, Benjamin, 1969.

[20] D. Ruelle, Superstable interactions in classical statistical mechanics, Commun. Math. Phys., 18 (1970), pp. 127-159. 